\title{
Tolerance to extreme doses of psychoactive substances
}

\section{DG Baitubayev ${ }^{1 *}$ and MD Baitubayeva²}

${ }^{1}$ Department of Psychiatric and Drug Addiction, Psychiatric Dispensary, Ridder City, Kazakhstan

${ }^{2}$ Semey State Medical University, Semey City, Kazakhstan

\section{Summary}

The article shows that the current level of physiology does not disclose the biological mechanisms of the organism's transition from one range to adapt to a higher one with an increase in the regular forces of the stimulus above sub-extreme. A new trend in the physiology of adaptation - proqredient adaptation, explains the mechanism of increasing the tolerance of the organism, with dependence on psychoactive substances (PAS). Scientific has reasonably shown that depending on the organism from PAS - not the disease, and the states like proqredient adaptation.

\section{The urgency of the issue}

It is known, that at the pick of dependence on any psychoactive substance (PAS), a person, for example, an opium (heroin) addict uses doses, which are multiple times, almost 10 times, higher than the lethal dose for an ordinary person [3,p.23].

The fact, that the drug user does not die, is explained by the increase in the body's tolerance in response to the increase in the dose of PAS [3,p.25].

Urgent issues of medicine are not only identification of mechanisms for increasing tolerance, but also a validation of the physiological process occurring on exposure to increasing doses of a psychoactive substance and the response increase intolerance of a PAS-dependent organism.

\section{Purpose and objectives of the study}

Adaptation reactions of the organism with regular exposure to a sub-extreme stimulus. Lack of adaptive reactions of the organism already known in physiology to explain the adaptation mechanisms in response to a further increase in the regular stimulus strength above the sub-extreme level in PAS-dependent patients.

The pronounced reactions by the vegetative nervous system (VNS) in PAS-dependent patients indicate the vegetotrophy of most of these substances. The amount of their influence is closest to the sub-extreme level. Responsive adaptive reactions of the organism under regular influence of the external factor of the average to sub-extreme strength were studied by L Kh Garkavi and co-authors (1977) [4,p.77]:

\section{More Information}

*Address for Correspondence: DG Baitubayev, Psychiatrist, Narcologist, Department of Psychiatric and Drug Addiction, Psychiatric Dispensary, Ridder City, Kazakhstan, Email: baitubayev@mail.ru; 02_madina@mail.ru

Submitted: November 27, 2021 Approved: December 30, 2021 Published: December 31, 2021

How to cite this article: Baitubayev DG, Baitubayeva MD. Tolerance to extreme doses of psychoactive substances. J Addict Ther Res. 2021; 5: 027-031.

DOI: 10.29328/journal.jatr.1001019

Copyright: @ 2021 Baitubayev DG, et al. This is an open access article distributed under the Creative Commons Attribution License, which permits unrestricted use, distribution, and reproduction in any medium, provided the original work is properly cited.

Keywords: Hypertrophy of the endocrine system; A state of regular, unfinished stresses; A progredient adaptation

D) Check for updates

O OPEn ACCESS

Under the influence of sub-extreme stimuli, an activation reaction with the stages of primary and persistent activation occurs, indicating a higher activity of protective systems. The stage of persistent activation is true, active resistance, stable and long enough - up to six months - in contrast to the training reaction and in the absence of constant exposure [4, p.79].

But in PAS dependence, the process does not result in the reaction of persistent activation; the dose to which the adaptation has occurred is habitual and results in no euphorizing effect.

To achieve a neurophysiological shift sufficient for euphoria, a larger dose is required.

But increase in the dose of PAS after the activation reaction is stressful for the organism. Stress in its development has three stages.

The first stage is the "anxiety reaction", the second one is the stage of tolerance when hypertrophy of the adrenal cortex with a steady increase in the formation and secretion of corticosteroids develops. They increase the amount of circulating blood and blood pressure, have an antihistamine effect, enhance gluconeogenesis, normalize physiological reactions, etc. The tolerance of the organism to the stimulus increases. Prolonged exposure to the stimulus results in the stage of exhaustion, and death may occur. Doses of PAS above the stress level are lethal [1]. 
L Kh Garkavi and co-authors showed that: "the reactivity of the organism is represented by a number of floors (ranges), which does not exceed ten. On each floor: a weak stimulus causes the training reaction, an average sub-extreme stimulus - the activation reaction, a strong stimulus - the stress. The ranges are separated by the zone of non-reactivity, when increasing the stimulus level above the stress one or decreasing below the training one causes no reaction. Transition to the next range shows again the same order of reactivity: the reactions of training, activation, and stress [4,p.77].

As it was mentioned above, an increase in the effect of an external stimulus up to a high level and the rise to the second stage of the stress reaction, the resistance stage, lead to the development of hypertrophy of the adrenal cortex with an increase in the secretion of corticosteroids increasing, in turn, resistance to the stimulus. But L Kh Garkavi and co-authors could not explain the mechanisms providing the human organism's transition (bypassing exhaustion and death stages) from one floor (range) of adaptation to a higher one, under the conditions of a further increase in the force of impact above stress level.

Indeed, according to pathophysiology, without such adaptation mechanisms increasing the tolerance, the body must die from "exhaustion", from the failure of adaptation mechanisms, when the organism transits from the first adaptation range to the second. But in PAS-dependent people, this is not observed. This indicates the failure of the current level of physiology to explain the mechanisms providing the body transition from one adaptation range (reactivity) to a higher one.

In the history of narcology, attempts were made to explain the increase in tolerance of organisms by different causes. They are accelerated disintegration of PAS in the addicts, development of chronic stress, activation of other states inactive in normal conditions, or activation of systems that fulfill other functions, but with an increase in a PAS, dose is forcedly involved in detoxification, etc. But all those assumptions have not been scientifically confirmed.

No matter how full modern scientific research explain qualitative changes at the cellular and molecular level that lead to an increase in tolerance in PAS dependent patients, it is clear that these changes can only be of adaptive, not pathological and damaging nature, otherwise, they would lead not to an increase intolerance, but rather to a decrease in it, and the body would already die when transiting from the first floor of adaptation to the second. Also, according to the dialectical principle of the mutual transition of qualitative changes to quantitative ones, the accumulation of these changes should lead to qualitative and quantitative changes in the neuroendocrine system which is responsible for the adaptation of the whole body.

I would like to quote L Kh Garkavi, EB Kvakina, MA Ukolova
(1977) - "it is possible to investigate separately the changes in any one system or at any one level, for example, molecular. But this is only a part of the changes in the overall complex reaction of the body".

Also, I would like to quote I N Pyatnitskaya (1988):

"Integral functional reactions to the intoxication of physiological systems are known to be no less important in maintaining homeostasis than biochemical protection" [5,p.58]. Consequently, we can speak about changes in the body's response to a drug".

\section{Object and methods of investigation}

Features of the response of the neuroendocrine system to any external stimulus. The capacity of the endocrine system for positive trophic changes. Hypertrophy and hyperfunction of the endocrine system - histological and biochemical evidence.

The response of the body to any change in the internal environment depends primarily on the functional state of the neuroendocrine system.

Thus, the cause of the altered body's reactivity and a steady increase in the overall tolerance of the body should be sought in the central mechanisms of adaptation - in the neuroendocrine system.

The increased intolerance of the PAS-dependent organism can be explained by the functional tension of the neuroendocrine system and by the reaction of persistent activation only within one adaptation range. It is good health, physical activity, increased protective capacities of the body to various hazards - hypothermia, etc., which are clinically observed in the prodroma and possibly in the initial stage of alcohol dependence.

But the tension in the neuroendocrine system and the reaction of persistent activation fail to explain the transition from a lower to a higher adaptation range following PAS exposure above sub-extreme level and its further increase! After all, in such a situation, the body must experience stress with exhaustion and death! This can only be explained by the transcendental functioning of the neuroendocrine system, which can be possible only due to its adaptive hypertrophy, in response to the regular exposure to the external factor. But is it possible? According to the theory of physiology of the development of interrelations between the structure and the function, in the course of ontogenesis (individual's development), functional activity is of particular importance and it is stimulated by the flow of stimuli affecting the organism as a result of changes in living conditions. Functional activity is the leading factor causing adaptive reactions in the body up to the development of morphological changes. Morphological changes occur in organs or systems stimulated by a flow of stimuli more regularly. 
Even in the early 1800s, J Lamarck suggested that "the work builds up the organs". P Lesgaft's merit was the explanation of a specific morphological alteration of the organism during the exercise process. V Ru showed that due to "trophic stimulation" in the working organ, the assimilation process begins to dominate over the dissimilation process, and morphological changes occur at the physiological level. The increase in energy reserves results in an increase in working efficiency.

It can be argued that the regular use of PAS - addressing the high response range - leads the entire body to the state of the activation reaction - hypermetabolic state, which does not contribute to the accumulation of reserves and the occurrence of positive trophic changes in the body. But one should remember that the hypermetabolic state develops in the "metabolic boiler" - at the level of tissue adaptation mechanisms [2,p.500]. Perhaps, in the higher adaptation mechanisms - the neuroendocrine system - despite their tension, there are no hypermetabolization processes, which contributes to the accumulation of reserves leading to morphological changes in the neuroendocrine system in the form of hypertrophy, is there?

The observations of L Kh Garkavi and co-authors indirectly prove possible accumulation of reserves in the neuroendocrine system during the activation reaction; "Although the metabolism is highly active during the activation reaction, it is characterized by an equilibrium" [4, p.79], since to ensure "equilibrium" of constantly growing metabolic processes, a "powerful" neuroendocrine system is necessary.

But in PAS dependence, after the completion of the activation reaction and in further enhancement of the stimulus above the sub-extreme level and transition to the subsequent adaptation floor, the "equilibrium" of the metabolic processes takes place, too. But this is possible only when the functional adequacy of the neuroendocrine systems grows in direct proportion to the strength of the external factor, which is possible only with the hypertrophic neuroendocrine system and, as a consequence, its hyper-productivity. The neuroendocrine system consists of the vegetative nervous system (VNS) and the endocrine part - the endocrine glands. In the functioning of the vegetative nervous system, a special mechanism is evolutionary provided that contributes to the accumulation of reserves - "advanced excitation" described in the 1930s by PK Anokhin.

The vegetative nervous system (VNS) responds to any stimulus with a somewhat excessive neurotransmitter ejection, as if in anticipation of possible future high consumption. VNS through neurotransmitters activates auxiliary and tissue adaptation mechanisms, and due to excesses of neurotransmitter ejection "takes a break" for its own recovery trophic processes. Although VNS regulates all the processes in the body, it has been established that there are biologically active substances produced by different cells of the body that have a trophic effect on VNS itself. One of such substances is the nerve growth factor (NGF) - an insulinlike substance that stimulates the growth of sympathetic ganglia. NGF is produced in the salivary glands by the smooth muscle fibers of the walls of internal organs. Similarly, the adaptive-trophic effect on VNS is provided by neuropeptides: liberins, somatostatin, enkephalins, endorphins, bradykinin, neurotensin, cholecystokinin, ACTH fragments, oxytocin [5,p.251].

"When excited in neurons, metabolic processes are intensified, the amount of RNA increases and the synthesis of proteins in neurons is enhanced. In neurons and glia cells surrounding them, these processes are multidirectional. RNA in nerve cells is increased due to the enhancement of its synthesis in a neuron and due to the transport of RNA from glial cells to neurons "[6,p.250].

But, despite the restorative and trophic processes, the vegetative nervous system cannot hypertrophy (the adrenal medulla is a modified sympathetic ganglion).

Adaptive, positive, trophic changes, during pauses, allow the sympathetic VNS just not to be exhausted, to maintain high activity for a long time - a kind of hyper functionality.

But there is no doubt that the mechanism of "advanced excitation" is also inherited in the endocrine part of the neuroendocrine system, when the endocrine glands, releasing excessive hormones, also "take a pause" for their own trophic recovery processes, but unlike the VNS, these processes lead them to hypertrophy and hyper-productivity. After all, hypertrophy resides in structures. Histological evidence of the endocrine system hypertrophy with regular exposure to a medium-strength stimulus is Selye's stress research: "adrenal glands bloom".

Speaking of adrenal hypertrophy, one should mean the adrenal cortex. As early as in the 1930s, it was found that chronic morphinization causes hypertrophy of the cortical layer of the adrenal glands in rats, which produces the "adaptation hormones" - glucocorticoids (hydrocortisone, cortisone and corticosterone), increasing the tolerance of the organism to intensive stimuli [3,p.260]. There is no doubt that hypertrophy of the adrenal cortex begins already during the activation reaction since the process of adrenal hypertrophy is not an abrupt process. There is no doubt that due to the mechanism of "advanced excitation" other endocrine glands also "take a pause" for trophic recovery processes, which leads to their hypertrophy and hyper functionality. Evidence of adaptive hypertrophy of the endocrine system is $\mathrm{L} \mathrm{Kh}$ Garkavi, and co-authors' observations under conditions of training and activation reactions - enlargement of the thymus gland and adrenal cortex, a prolonged increase in the thyroid and reproductive gland functions [4,p.78].

\section{Results and discussion}

Thus, under the regular exposure to PAS as a sub-extreme 
stimulus, while hypermetabolic processes occur in the "metabolic boiler," accumulation of reserves takes place in the endocrine system, as a result of "advanced excitation". This accumulation of reserves leads to adaptive hypertrophy and hyperfunction of the endocrine system, which results in an increase in the tolerance of the body.

That is why, a subsequent, increasing, potentially extreme dose of PAS has a sub-extreme non-pathogenic effect on the body. The condition persists for further adaptation (Figure 1).

Thus, in PAS dependence, in each range and in the transition to a higher adaptation range, one should speak not of the reaction dyad: activation and persistent activation, but of the reaction tetrad: activation, persistent activation, then stress with "anxiety reaction", and the stage of resistance. And the hypertrophy of the endocrine system that has developed to this moment, does not allow the development of the final stage of stress - exhaustion. At this moment, the zone of non-reactivity separating the ranges is observed, when the intensification of the stimulus above the stress level or the weakening below the training one does not cause any reaction. With an increase in the dose of PAS and transition to another range, everything comes around. It is more correct to call such a process not a state of chronic stress, but a state of regular, unfinished stress. Stress without the exhaustion stage, no matter how regular it is, cannot be considered a disease. That allows the body to transit to a higher range of adaptation without death. It becomes clear that increased resistance in persistent activation reaction responding to regular sub-extreme exposure to PAS and the resistance stage in stress reaction responding to the further regular exposure to an increasing dose of PAS are functional manifestations of adaptive hypertrophic changes in the endocrine system.

This process is called progredient (progressive) adaptation. Beliefs about the unity of form and function, the stereotyped thinking that "if changes in the body are acquired

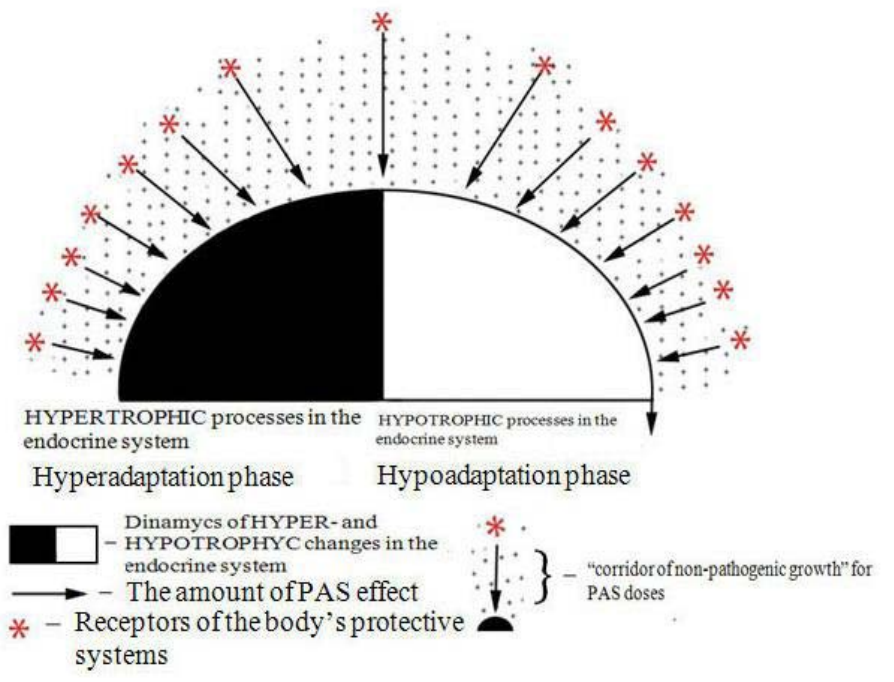

Figure 1 and irreversible, therefore, they are pathological," have led to the erroneous judgment that the body's dependencies on PAS should be considered as diseases. There is the expression "any disease is an adaptation." But the opposite statement that "an adaptation is a disease" in relation to PAS dependencies is inadmissible. After all, hypertrophy of the endocrine system, its high adaptation adequacy, leading to an increase in general and specific resistance, do not result in failure or lack of adaptive capabilities. Consequently, there is no need to compensate for the adaptive capacity of the body at the expense of the tissues and the systems of the body - the disease does not arise. Thus, an increase in adaptive capacity is directly proportional to the increase in a dose; the role of receptors of the body's protective systems ${ }^{*}$ - in the figure) indicating possible PAS overdose, the experience of narcotization are also important.

Due to the vegetotrophic nature of PAS, in PAS dependencies, in contrast to the disease, the mechanism of PAS action is also different. Common pathogenic factors - without pronounced vegetotrophy - cause damage at first, and only later a protective reaction of the organism develops. PAS, simultaneously or primarily, affects the receptors of VNS, which causes its timely or even advanced reaction to possible damage from the PAS effect. In PAS dependence, the increase in the exposure dose occurs through the "non-pathogenic corridor" - between the signals of the body's protective systems - receptors indicating a possible overdose, and the hypertrophying endocrine system (Figure 1). That is why the acquired biological changes in PAS dependence are only of adaptive nature. And thereby, one should talk about the adaptive attraction, adaptively changed behavior, adaptively changed reactivity to PAS, or, conversely, about the readaptation - deprivation syndrome, and so on.

In the final stage of the dependence, depletion of the adaptive capabilities of the organism, due to the hypotrophy of the endocrine system, the receptors of the body's protective systems indicate possible PAS overdose. This leads to a parallel decrease in the dose of PAS that a person is able to adapt to, the effect of PAS turns out to be sub-extreme again, and pathology is not observed either (Figure 1).

Pathology in PAS dependence is an accompanying phenomenon.

The explanation of the increased tolerance by means of the adaptive hypertrophy of the endocrine system does not contradict the development of biochemical theories of the euphoria etiology and explains the internal mechanism of clinical manifestations in PAS dependence.

Under alcohol exposure, when abuse with adaptive, qualitative, or quantitative changes in the mechanisms responsible for the euphoria is required to achieve euphoria and acquire dependence, the accompanying increase intolerance can be explained by the endocrine system hypertrophy.

The VNS activity and productivity of the hypertrophying 
endocrine system in the first stage of alcohol dependence explain maintaining the body tone during the week intervals of sobriety, in the absence of alcohol stimulation.

Compensatory stress of VNS and sufficient production of neurotransmitters or residual neurotransmitter excess, explain adrenergic tension and expressed vegetative disorders in the alcohol withdrawal syndrome.

The activity of the sympathetic part of VNS against the background of the gradual exhaustion of the parasympathetic department (the adrenergic system is more stable in ontogenesis, too) also explains the qualitative change (according to narcotism age) of the sedative PAS (hypnotics, alcohol, opiates) effect on the body, transformation of their initial sedative action into a stimulating one.

The hypertrophy of the endocrine system due to prior narcotization (and hence an increase in overall resistance) explains the rapid development of alcohol dependence in former opium addicts in alcoholization: rapid increase in alcohol tolerance, the rapid formation of alcohol abstinence syndrome, the development of binge drinking (to develop alcoholism in former drug addicts, it is sufficient to develop only a specific tissue adaptation to alcohol).

Stimulation of the hypertrophic endocrine system and increase in the overall resistance of the organism explain the fact that many stimulants (caffeine), eliminating some effects of ethanol, however, do not change its pharmacokinetics, but prolong its intoxicating effect.

Initially, the psychomotor agitation in PAS-dependent people getting narcosis can be based on the excitement of the active, sympathetic department of VNS.

As the PAS dependence develops, due to the mechanism of "advanced excitation", contributing to adaptive activityhyper functionality of the sympathetic VNS, the hypertrophy and hyper-productivity of the endocrine system lead to the fact that the role of the entire neuroendocrine system as a functional mechanism of protection and adaptation increases and becomes the leading one.

\section{Conclusion}

1. Under regular sub-extreme exposure of the organism to a psychoactive substance, the mechanism of "advanced excitation" allows maintaining the activity of the sympathetic VNS, leading to hypertrophy of the adrenal cortex.

2. Under regular sub-extreme exposure to a psychoactive substance, adaptive maintenance of sympathetic VNS activity and adaptive hypertrophic changes in the endocrine system lead to an increase in the tolerance of the body.

3. In psychoactive substances dependence, due to the adaptive activity of the sympathetic VNS, adaptive hypertrophy and hyper-productivity of the endocrine system, potentially extreme doses have a nonpathogenic sub-extreme effect on the human organism.

4. Dependence of the body on psychoactive substances due to the increased tolerance of the organism and the transformation of the effect of potentially extreme doses into the sub-extreme effect is the adaptation process.

Recommendation: It is necessary to validate the dependence of the body on psychoactive substances not as a disease, but as a state of progredient adaptation.

\section{References}

1. Zaichik AS, Churilov LP. General pathophysiology. St. Petersburg. 2005; 525: 23-26.

2. Korobkov AV, Bashkirov AA, Vetchinkina KT. Normal physiology. Chapter 28 Physiology of adaptation. Moscow: High School, 1980; 560: 499.

3. Pyatnitskaya IN. Clinical Drug Addiction. Moscow: Medicine, Leningrad branch. 1975. 333: 3-6; 22-45.47-55; 255-256, 261 -263; 268-274.

4. Pyatnitskaya IN. Alcohol abuse and the initial stage of alcoholism. Moscow: Medicine. 1988. 286: 77-80.

5. Smirnov VM, Yakovlev VN. Physiology of the central nervous system. Moscow: Academy. 2004. 184-185; 250-251.

6. Kirillov OI. Stress hypertrophy of the adrenal glands. Moscow: Science. 1994; 176. 